\title{
Subjet Distributions in NC DIS
}

\section{Elias Ron (Universidad Autónoma de Madrid, Spain)}
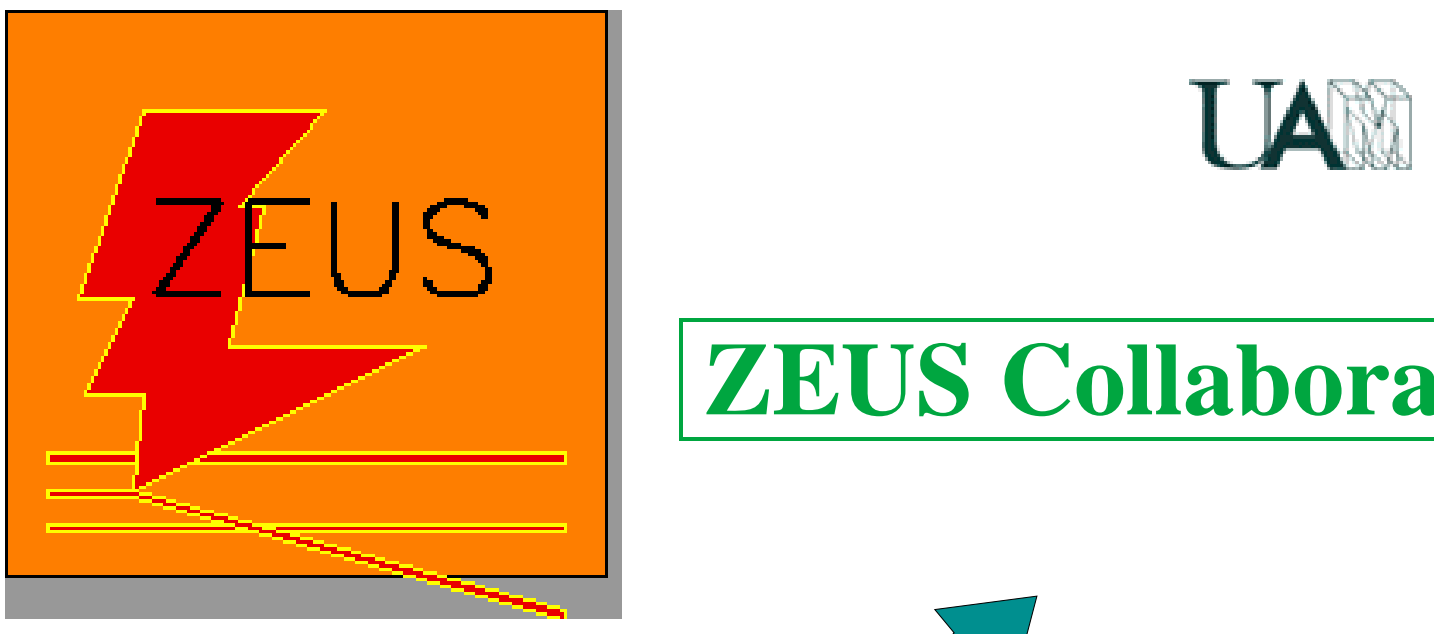

$\mathrm{e}(\mathrm{Ee}=27.5 \mathrm{GeV})$

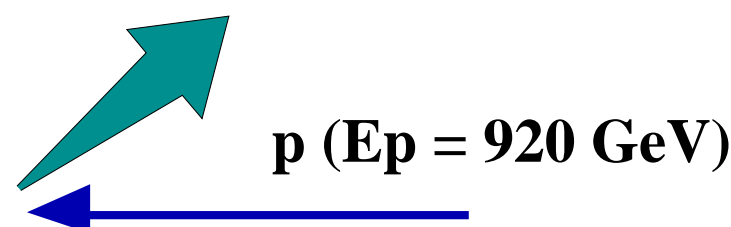

\section{ZEUS Collaboration}

$$
\sqrt{\mathrm{s}}=318 \mathrm{GeV}
$$

HERA

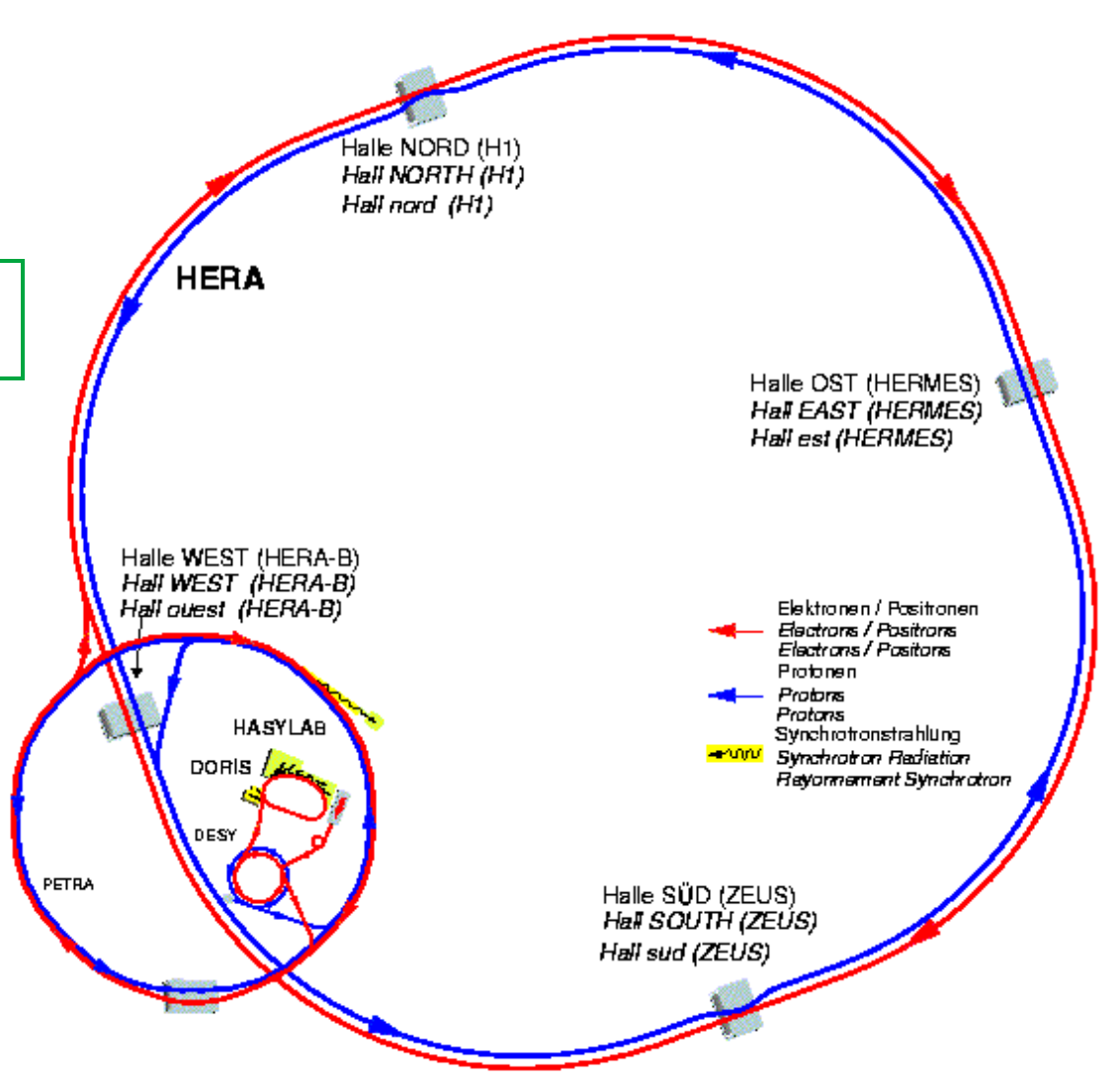




\section{Internal Structure of Jets}

- The investigation of the internal structure of jets gives insight into the transition between a parton produced in a hard process and the experimentally observable jet of hadrons.

- At sufficiently high $E_{T}^{j e t}$, the internal structure of jets is expected to be calculable in pQCD, since the fragmentation effects are small.

- Parton radiation is described in pQCD by means of the splitting funtions $P_{a b}(x, \mu)$, which give the probability of a parton $b$ arising from a parton $a$ with a fraction $x$ of its momentum.

- $O\left(\alpha_{s}^{2}\right)$ calculations can be obtained in the laboratory frame with up to have 3 partons in one jet, which corresponds to the NLO contribution to substructure.
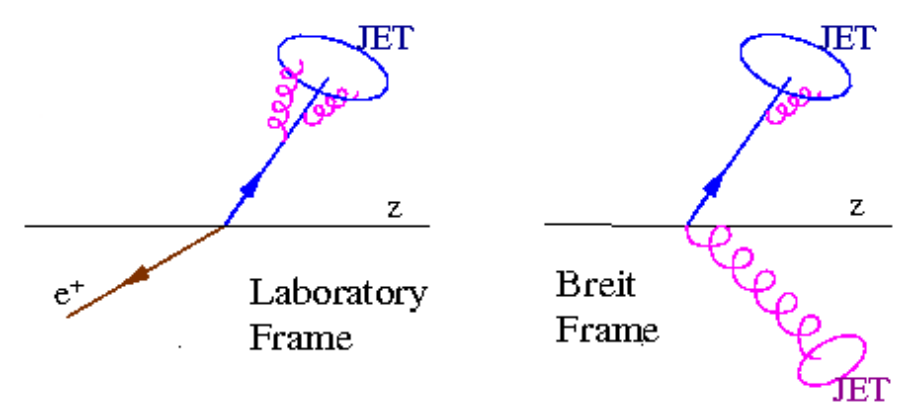
- The internal structure of jets can be studied my means of subjets.

- Subjets are obtained within a jet by reapplying the $k_{T}$ cluster algorithm on all the particles belonging to the jet, until for every pair of clusters the distance between them is greater than $d_{c u t}$, with

$d_{c u t}=y_{c u t}\left(E_{T}^{j e t}\right)^{2}$

$\boldsymbol{y}_{\text {cut }}=$ resolution parameter

- The remaining clusters are called subjets.

- The subjet multiplicity depends on the value of $y_{c u t}$

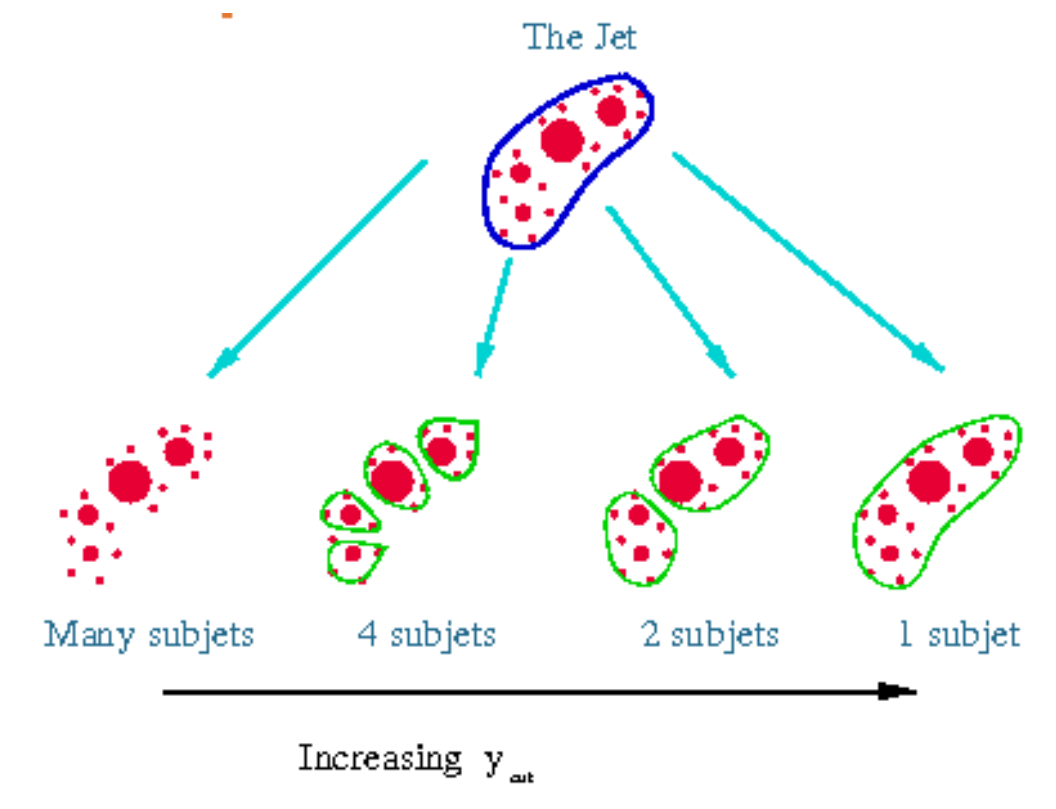


-In this analysis, jets were reconstructed in NC DIS events with $Q^{2}>125 \mathrm{GeV}^{2}$

- The jets must satisfy:

$E_{T}^{j e t}>14 \mathrm{GeV}$ and $-1<\eta^{j e t}<2.5$

- We studied in detail the pattern of QCD radiation from a primary parton by measuring normalised cross sections as a function of the subjet observables in two different jet samples.

- The first sample corresponds to jets with exactly two subjets at a value of $y_{c u t}=0.05$. (82 $p b^{-1}$ of ZEUS data)

- The second sample corresponds to jets with exactly three subjets at a value of $y_{c u t}=0.03$. (334 $p b^{-1}$ of ZEUS data)
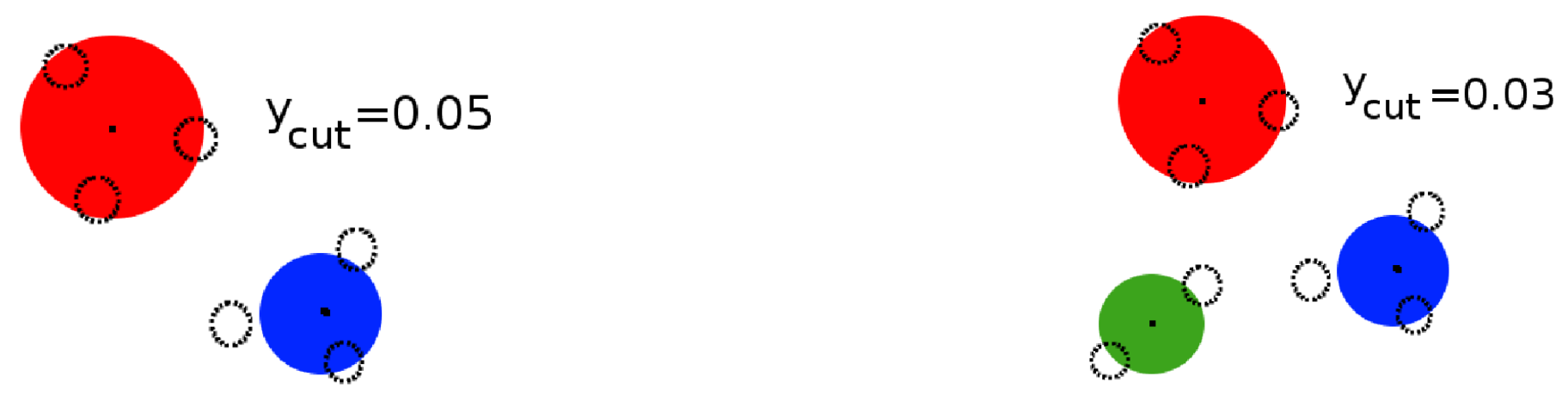
- Measurements of normalised cross sections as functions of observables sensitive to the pattern of parton radiation:

$$
E_{T}^{s b j} / E_{T}^{j e t}, \eta^{s b j}-\eta^{j e t},\left|\phi^{s b j}-\phi^{j e t}\right| \text { and } \alpha^{s b j}
$$

and their variation with the scale by studying the dependence with:

$$
E_{T}^{j e t}, Q^{2} \text { and } x_{B J}
$$

- The value of $y_{c u t}=0.05$ and $y_{c u t}=0.03$ chosen are a compromise between statistics,resolution and hadronisation corrections.

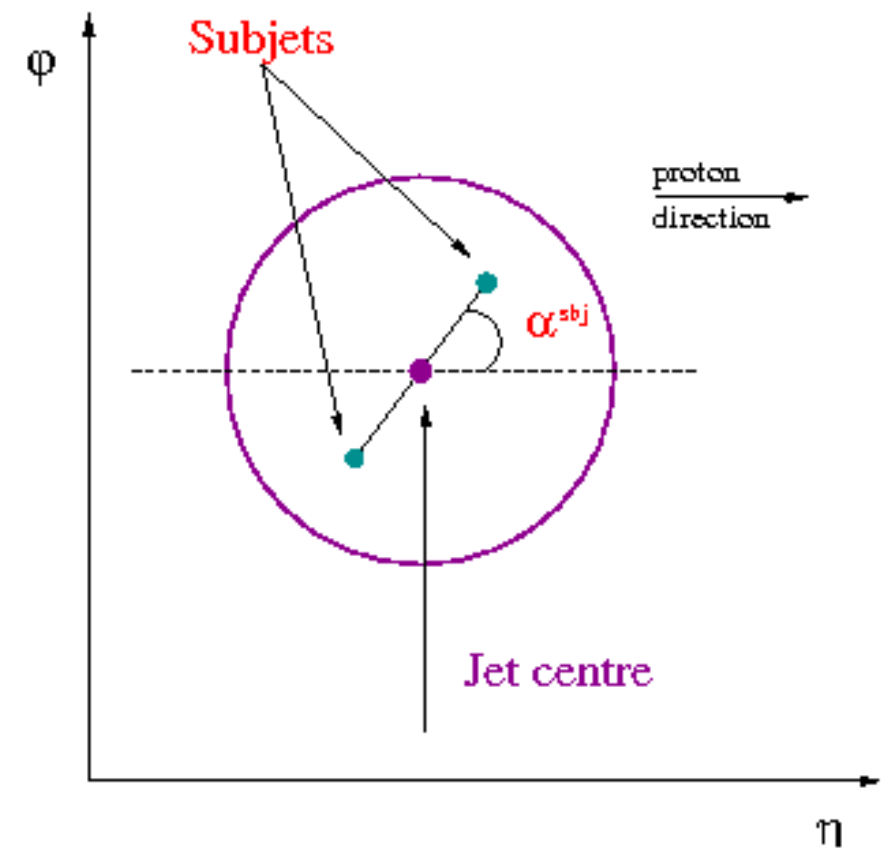




\section{DATA vs NLO: RESULTS}

\section{ZEUS}

Two-subjet jets

- Normalised cross sections for:

- $E_{T}^{s b j} / E_{T}^{j e t}$

$-\eta^{s b j}-\eta^{j e t}$

$-\left|\phi^{s b j}-\phi^{j e t}\right|$

$-\alpha^{s b j}$

- NLO calculations give an adequate prediction of the data
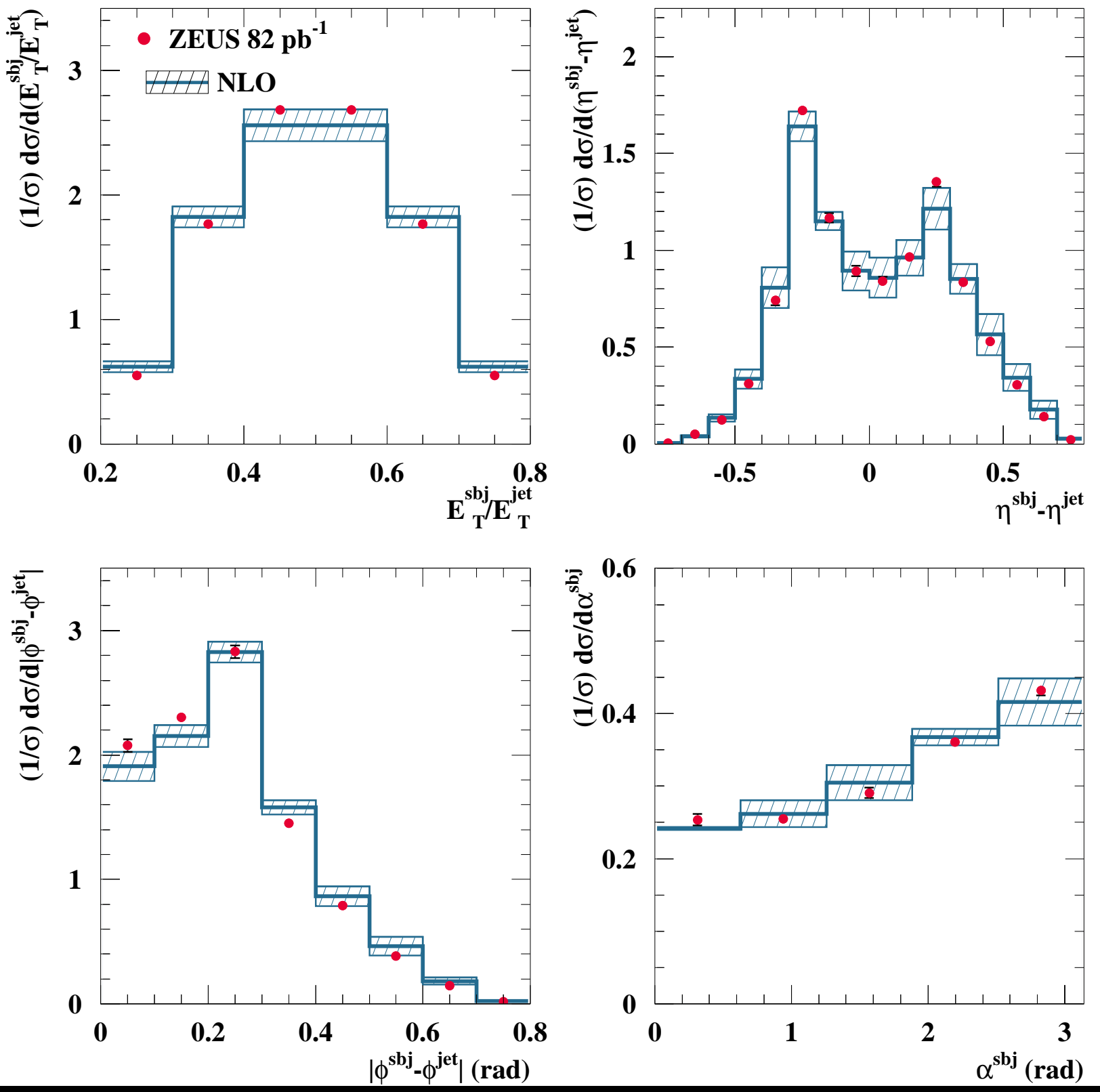

\section{Elias Ron (UAM)}


- We also study the coherence effects between initial and final states parton radiation.

- Soft emissions (low- $E_{T}$ subjets) will tend to be in the

\section{ZEUS} direction of the proton beam.

- Highest $E_{T}$ subjet expected to be in the rear part of the jet.

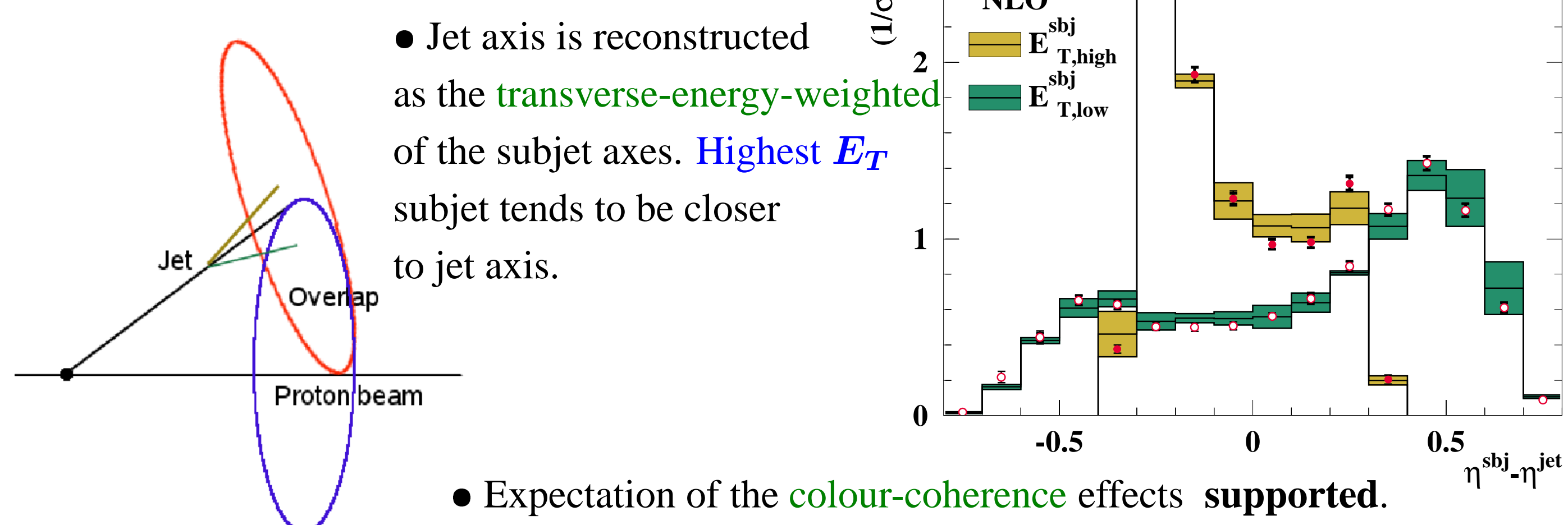


ZEUS

- Normalised cross sections for:

$-E_{T}^{s b j} / E_{T}^{j e t}$

$-\eta^{s b j}-\eta^{j e t}$

$-\left|\phi^{s b j}-\phi^{j e t}\right|$

$-\alpha^{s b j}$

versus the gluon- and quark- induced processes separately.

- The $\boldsymbol{O}\left(\boldsymbol{\alpha}_{S}^{2}\right)$ prediction: $82 \%$ of q-induced and $18 \%$ of g-induced

- The data are better described by the prediction of the q-induced processes.
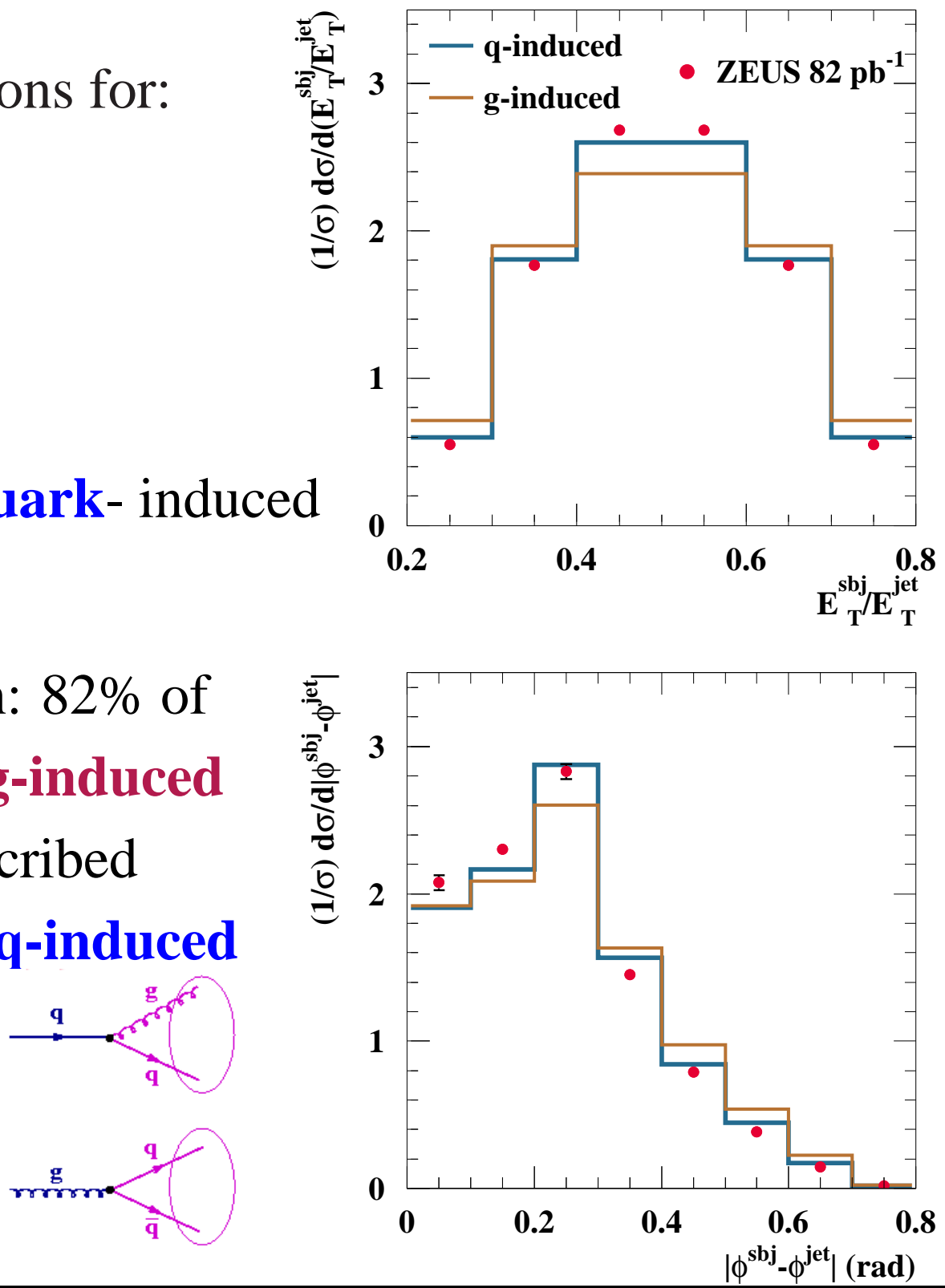
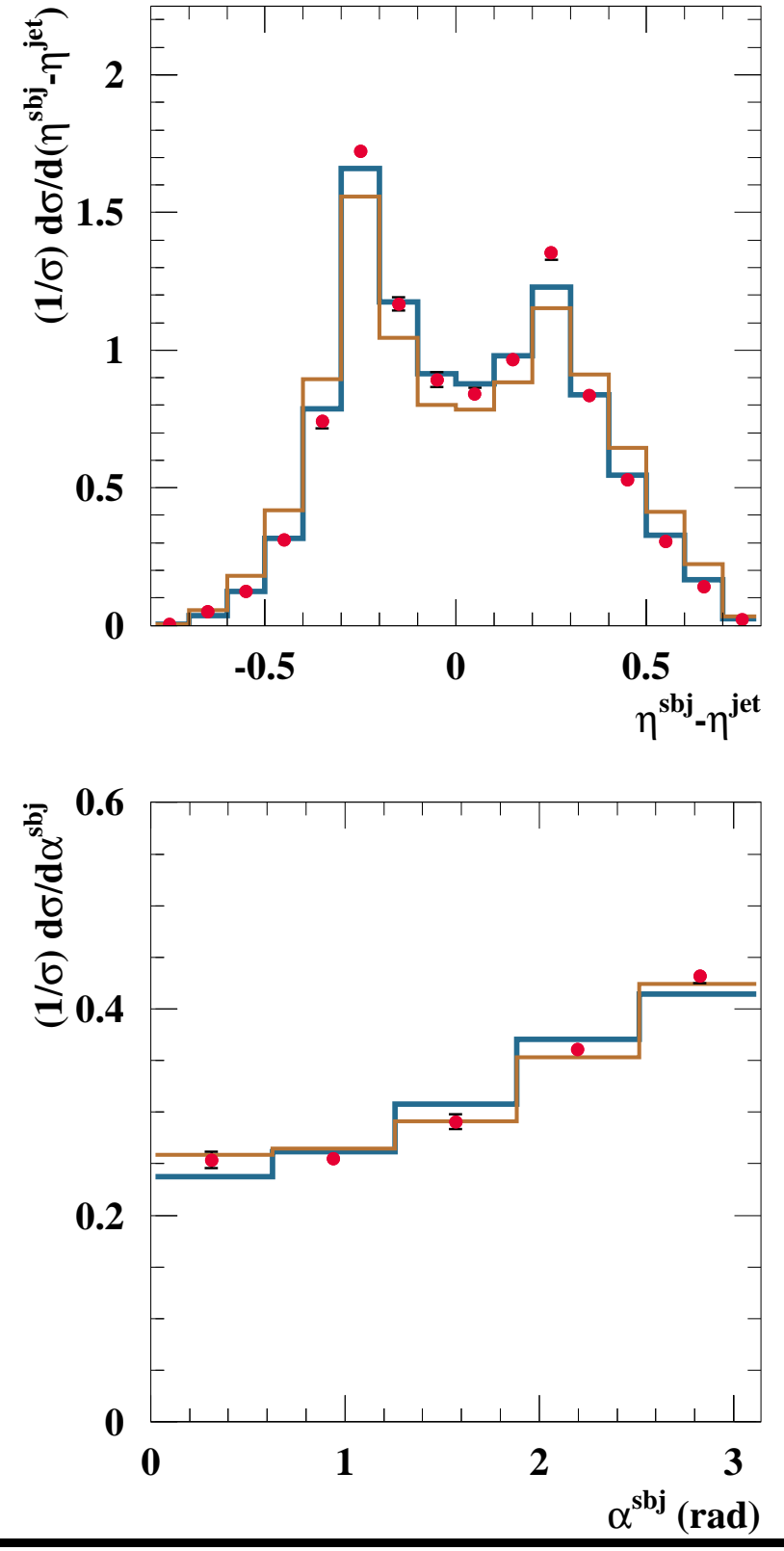


\section{DATA vs NLO: RESULTS}

\section{ZEUS}

Two-subjet jets

Dependence with $\boldsymbol{E}_{\boldsymbol{T}}^{j e t}$

- Data have similar shape for all $\boldsymbol{E}_{\boldsymbol{T}}$ regions, which agrees with the expected scaling behaviour of the splitting functions.

- These features are reasonably reproduced by the NLO calculations.

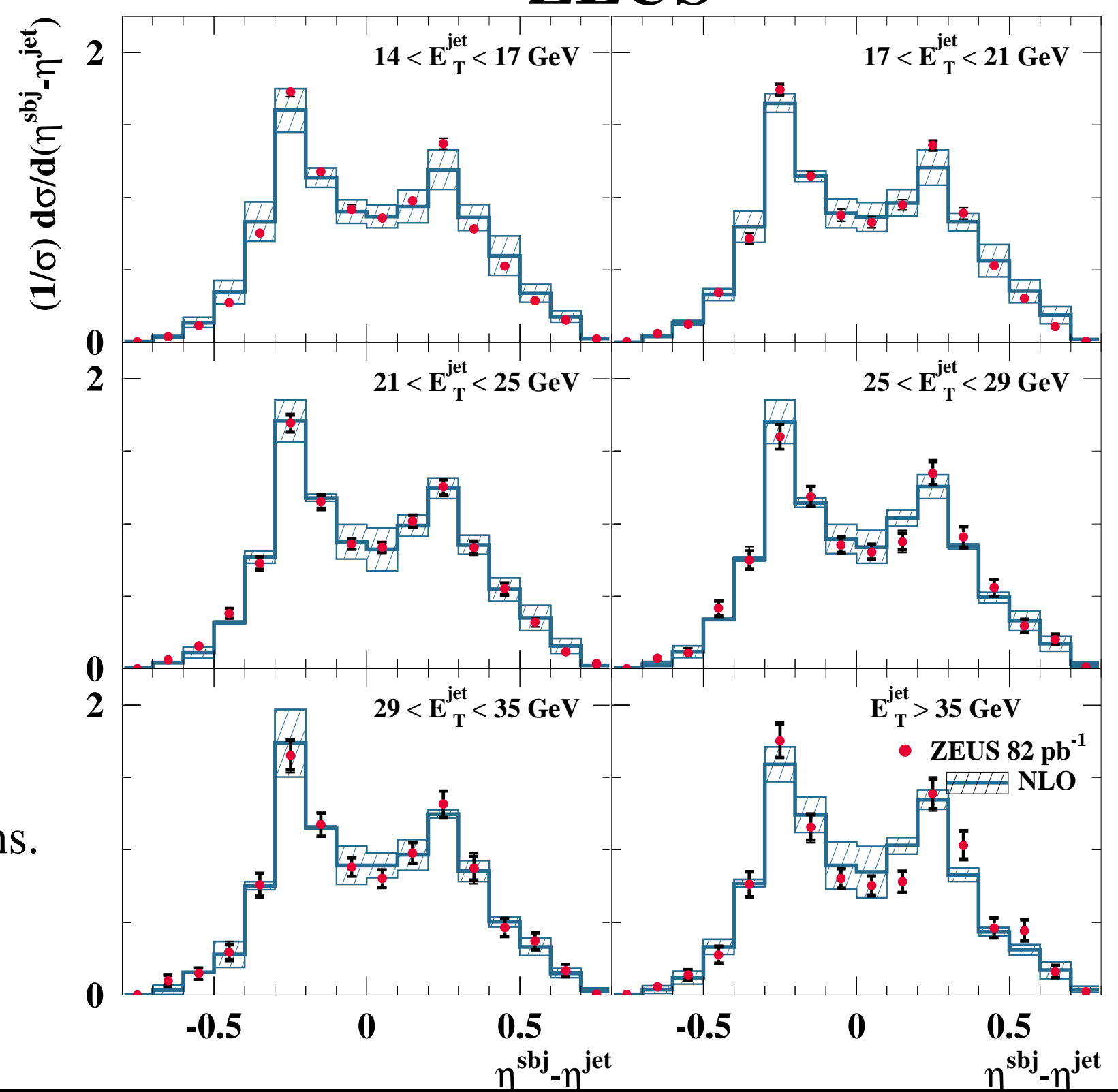




\section{DATA vs NLO: RESULTS}

\section{ZEUS}

Two-subjet jets

Dependence with $\boldsymbol{E}_{\boldsymbol{T}}^{j e t}$

- Data have similar shape for all $\boldsymbol{E}_{\boldsymbol{T}}$ regions, which agrees with the expected scaling behaviour of the splitting functions.
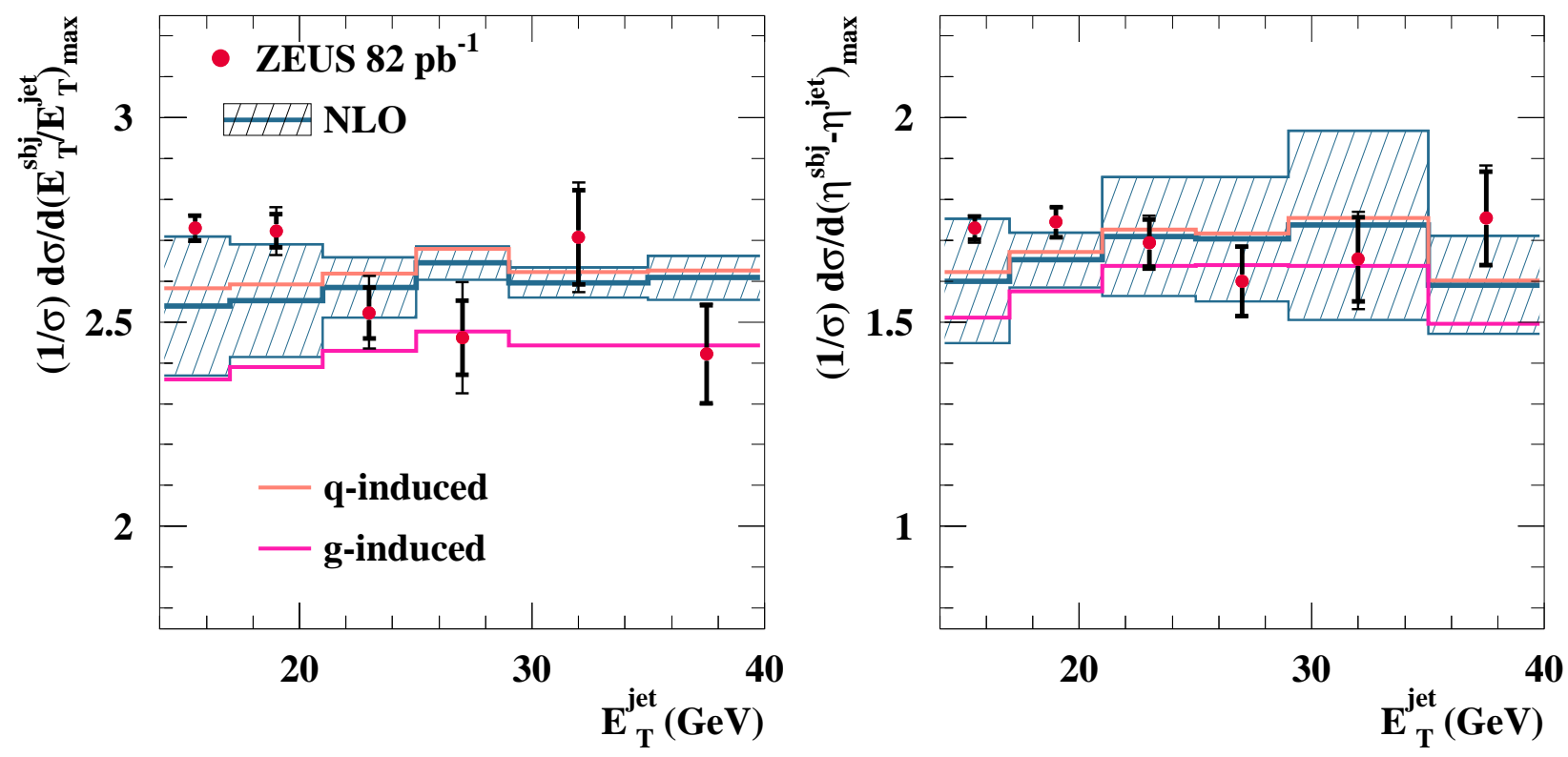

- These features are reasonably reproduced by the NLO calculations.
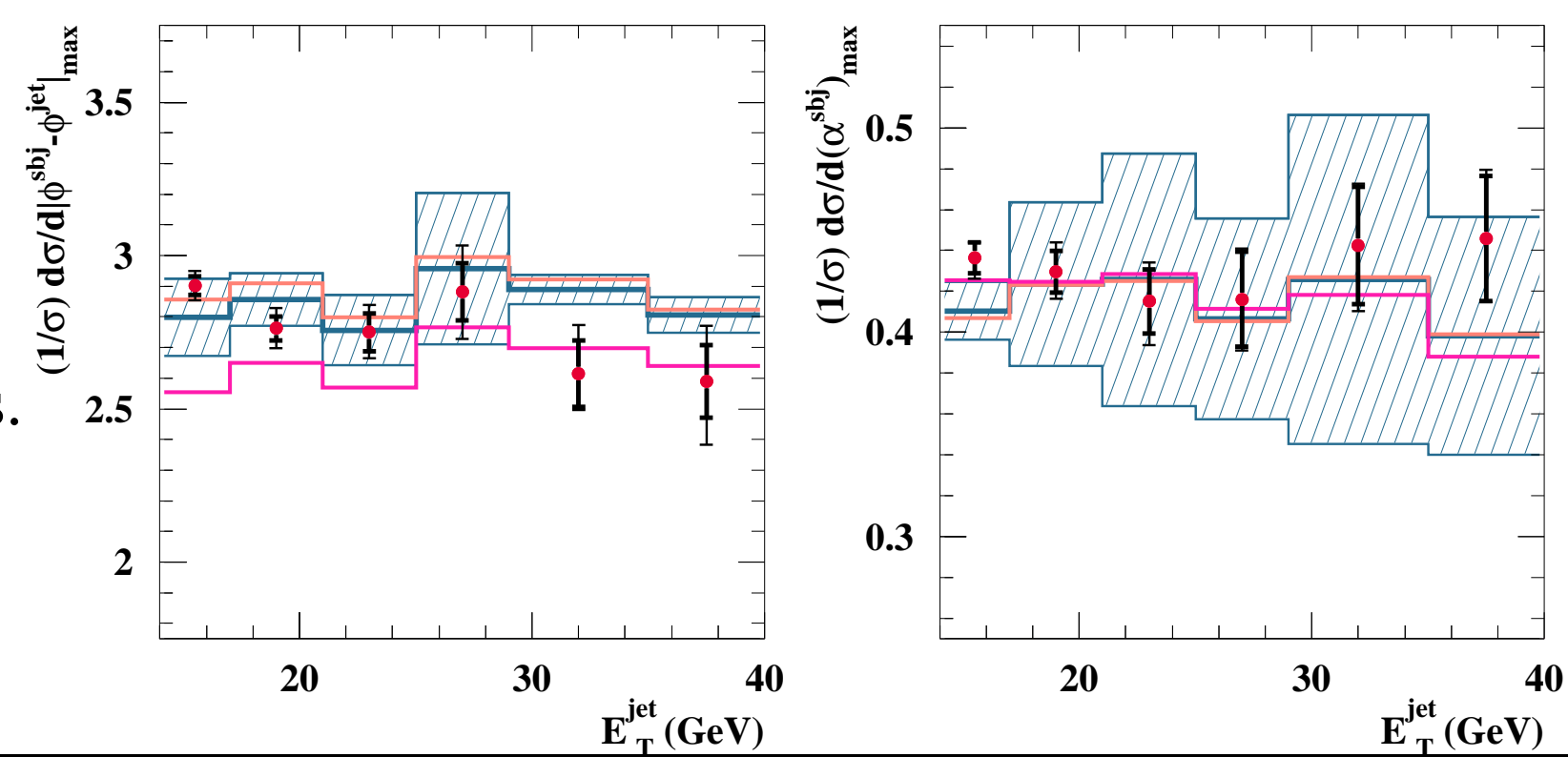

\section{Elias Ron (UAM)}




\section{DATA vs NLO: RESULTS}

\section{ZEUS}

Two-subjet jets

Dependence with $Q^{2}$

- fraction of gluon-induced events varies from $32 \%$ in the first bin to $14 \%$ in the other regions.

- At low $Q^{2}$ scaling violations are more prominent.

- These features are reasonably reproduced by the NLO calculations.
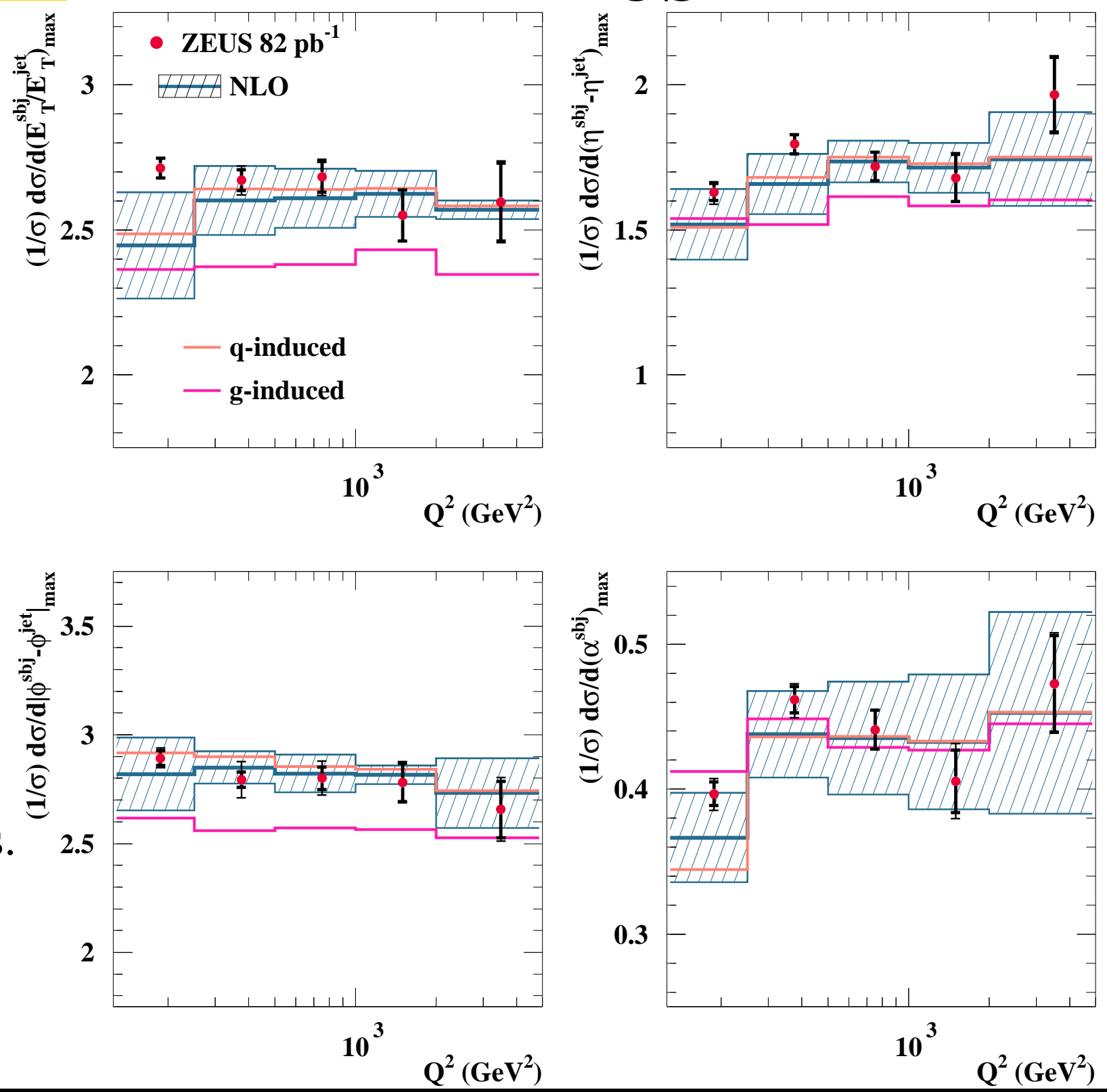


\section{DATA vs NLO: RESULTS}

\section{ZEUS}

Two-subjet jets

Dependence with $x_{B J}$

- Data have similar shape for all $\boldsymbol{x}_{\boldsymbol{B} \boldsymbol{J}}$ regions, which agrees with the expected scaling behaviour of the splitting functions.
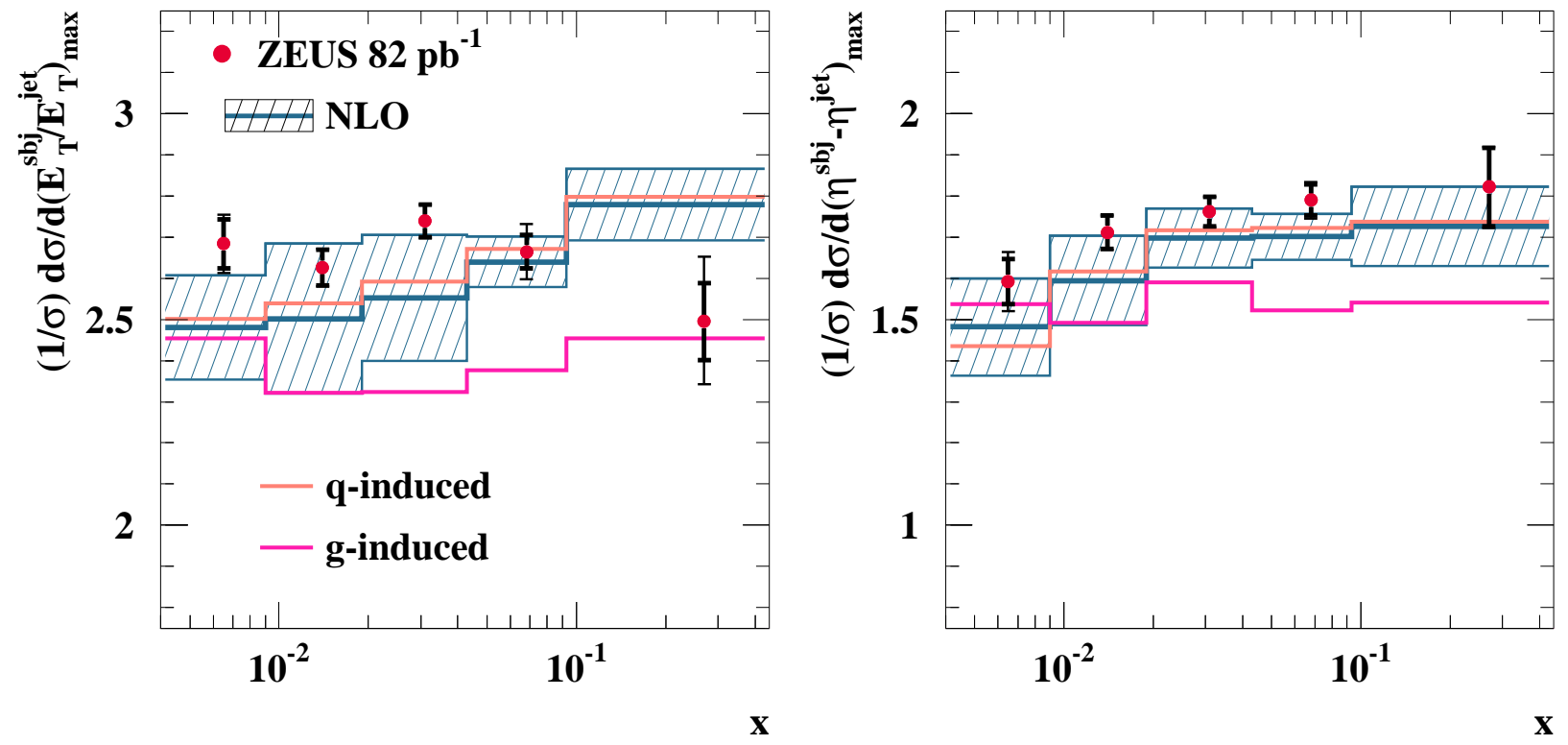

- At low $\boldsymbol{x}_{\boldsymbol{B} \boldsymbol{J}}$ scaling violations are more prominent.

- Similar features as in $\boldsymbol{Q}^{\mathbf{2}}$
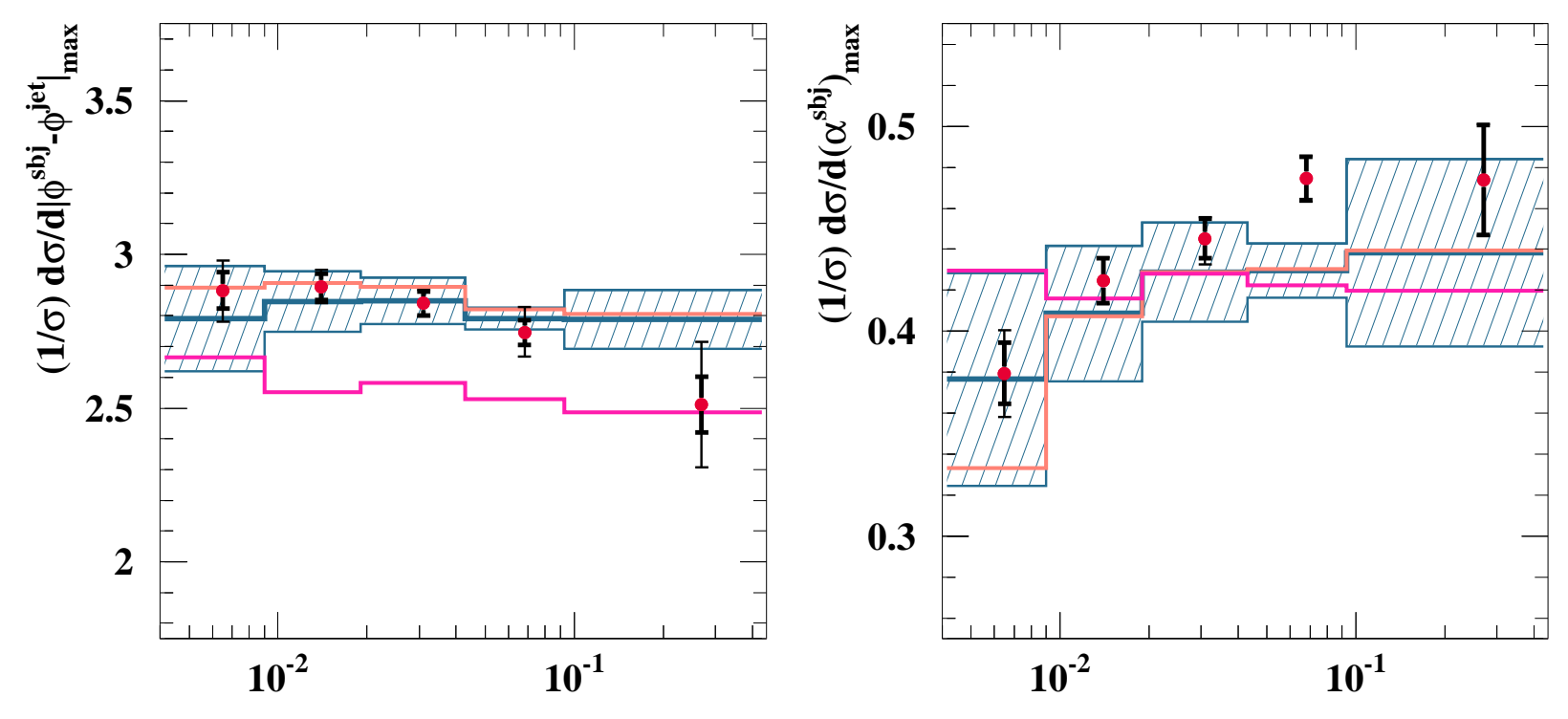


\section{Three-subjet jet structure}

- We now extend our studies of jet substructure by measuring subjet cross sections with respect to the variables:

$\rightarrow \alpha^{s b j}$ : The angle, as viewed from the jet centre in the $\eta-\phi$ plane, between the lowest $E_{T}$ subjet and the proton beam direction.

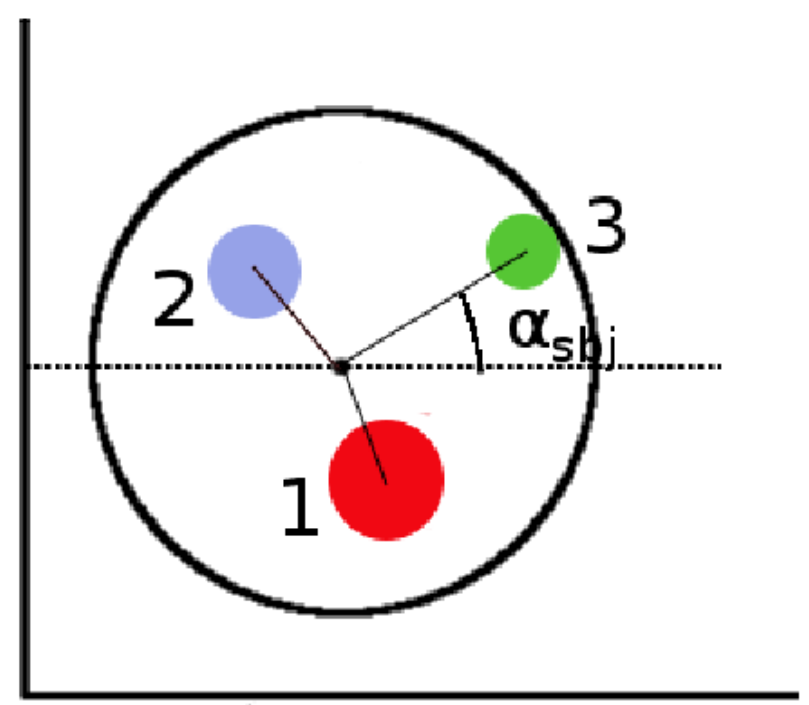




\section{DATA vs LO: RESULTS}

- Normalised cross sections for:

$\alpha^{s b j}, \eta_{\text {high }}^{s b j}-\eta^{j e t}, \eta_{\text {low }}^{s b j}-\eta^{j e t}$

Three-subjet jets at $\boldsymbol{y}_{\text {cut }}=\mathbf{0 . 0 3}$

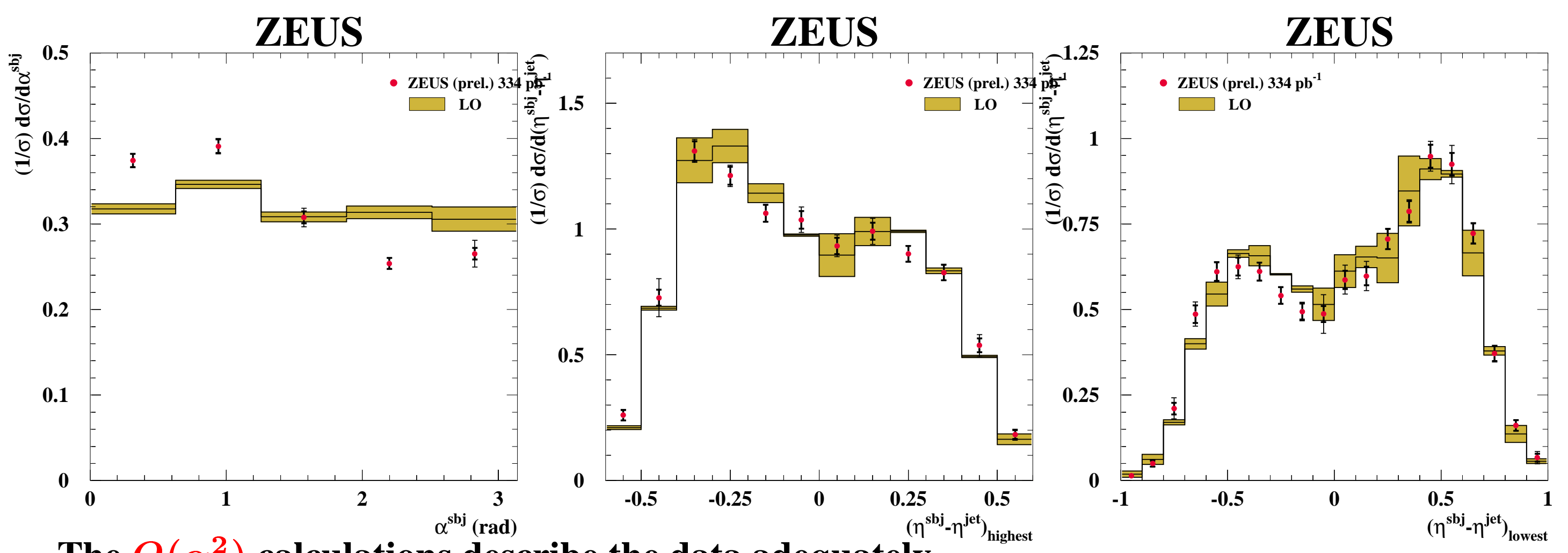




\section{CONCLUSIONS}

- The data show:

-Subjets tend to have similar transverse energies.

-The lowest $\boldsymbol{E}_{\boldsymbol{T}}$ subjet tends to be in the forward direction.

This supports the presence of colour coherence effects

between inital and final states.

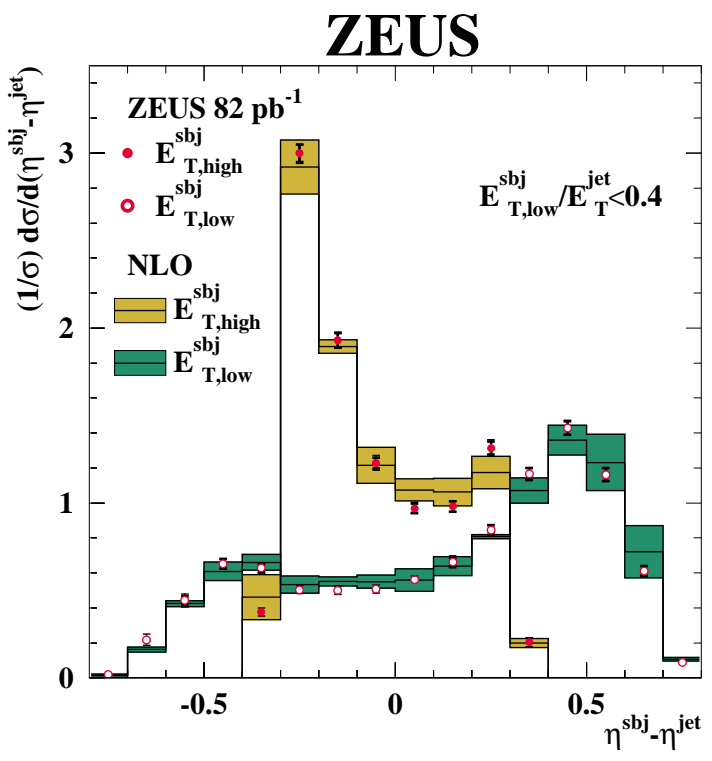

- In the two-subjet sample:

-A weak dependence on $\boldsymbol{E}_{\boldsymbol{T}}^{j e t}$ is observed, in agreement with the expected scaling behaviour of the splitting functions.

-At low $\boldsymbol{Q}^{2}$ and low $\boldsymbol{x}$ some differences are observed, which can be attributed to scaling violations.

- This features about the pattern of QCD radiation, as well as the evolution of the cross sections with the scale are reasonably well described by the NLO calculations.

- As well, the data are better described by the calculations for jets arising from a quark-gluon pair. 